\title{
Mechanics of inhomogeneous turbulence and interfacial layers
}

\author{
By J. C. R. HUNT $T^{1}$, I. EAMES AND J. WESTERWEEL $\mathrm{L}^{3}$ \\ ${ }^{1}$ Department of Earth Sciences, University College London, Gower Street, \\ London, WC1E 6BT, UK \\ ${ }^{2}$ Departments of Mechanical Engineering and Mathematics, University College London, \\ Torrington Place, London, WC1E 7JE, UK \\ ${ }^{3}$ J. H. Burgers Centre, Delft University of Technology, Leeghwaterstraat 21, \\ 2628 CA Delft, The Netherlands
}

(Received 10 July 2005 and in revised form 31 January)

The mechanics of inhomogeneous turbulence in and adjacent to interfacial layers bounding turbulent and non-turbulent regions are analysed. Different mechanisms are identified according to the straining by the turbulent eddies in relation to the strength of the mean shear adjacent to, or across, the interfacial layer. How the turbulence is initiated and the topology of the region of turbulence are also significant factors. Specifically the cases of a layer of turbulence bounded on one, or two, sides by a uniform and/or shearing flow, and a circular region of a rotating turbulent vortex are considered and discussed.

The entrainment processes at fluctuating interfaces occur both at the outer edges of turbulent shear layers, with and without free-stream turbulence (e.g. jets, wakes and boundary layers), at internal boundaries such as those at the outside of the non-turbulent core of swirling flows (e.g. the 'eye-wall' of a hurricane) or at the top of the viscous sublayer and roughness elements in turbulent boundary layers. Conditionally sampled data enables these concepts to be tested. These concepts lead to physically based estimates for critical modelling parameters such as eddy viscosity near interfaces, entrainment rates, maximum velocity and displacement heights.

\section{Introduction}

In the past 50 years (1955-2005), the main development in turbulence research has been the measurement, computation and analysis of the different types of coherent eddy motions and their distributions, especially in inhomogeneous flows (Holmes, Lumley \& Berkooz 1996). General concepts derived from these studies have helped to provide physically plausible explanations of the quantitative statistical results obtained by Kármán, Prandtl, Taylor, Richardson, Kolmogorov and Obukhov in the previous 40 years (1915-1955). In fact Richardson (1922), Prandtl (1925) and Kolmogorov (1941) themselves used explicit models of eddy motion to justify and illustrate their ideas. Quantitative experimental studies of eddy motion only became possible through the use of conditional sampling and improved flow visualization methods (e.g. Hussain 1986). These data lead to dynamical studies of the eddies in turbulent flows as they move individually and interact collectively (Hunt \& Vassilicos 2002). The results of the research on eddy motions have greatly enriched the subject, especially for the non-specialist through the description and understanding of many 
of the qualitative features of turbulence, both those observed in nature and those resulting from human activities ranging from engineering, to cooking and to the fluid motions of our own bodies (e.g. Lugt 1983). Many practical benefits have resulted from this greater understanding in every branch of engineering and some branches of medicine (e.g. Hunt 1985; Pedley 1980).

The Journal of Fluid Mechanics has played an important role in encouraging and publicizing complementary ways of studying both homogeneous and inhomogeneous turbulence. Some of the papers were quite controversial (e.g. Chandrsuda et al. 1978) and in one case involved not only many more than the usual number of referees in the editorial process, but also an advisory footnote about the paper (Long \& Chen 1981)! New and tentative results were published and topical conferences were reviewed. The old flimsy issues of JFM used to be passed around laboratories and discussed in tea rooms and lunch queues, as they were at the Central Electricity Research Laboratories (where J.C.R.H. was working 1968-1970). The equivalent discussions now happen on the internet in one form or another. In one issue in 1968 there were two papers on Professor Scorer's conjectures (which have still not been resolved) about the 'turbulent diffusion' of angular momentum in rotating flows. They demonstrated the value of simple experiments, one of which involved an Alka Seltzer tablet to produce the turbulence (Gough \& Lynden-Bell 1968). Bretherton \& Turner (1968) explained the importance of this for geophysical fluid mechanics.

There are still many fundamental and interesting problems in fluid mechanics which are not well understood. One hopes that researchers will continue to convey the excitement of these studies in their JFM papers as well in articles in the popular scientific press such as Nature, New Scientist, etc. Simple theoretical and experimental methods are still necessary, but we now have the great advantage of using detailed numerical computation and three-dimensional velocity measurement systems on scales from the microscopic to the galactic. These techniques enable many aspects of the flow to be described, including variability over large numbers of similar events. Sometimes they have confirmed the imaginative predictions of earlier generations of fluid mechanicists (for example the form of cyclonic storms first depicted in the 1860s correspond very closely to the satellite pictures of the 1950s). On other occasions, the new data have given rise to surprises; the example given in this paper is the sharpness of turbulent interfaces.

Here we review the aspects of eddy motion that occur at boundaries between regions of turbulent and non-turbulent motion such as the outer edge of jets and wakes. These regions of very inhomogeneous turbulence play a critical role in many engineering and natural flows that is now beginning to be appreciated (e.g. Hunt $e t$ al. 2001). In some previous models it was assumed that the dynamics of the interface was determined by the 'nibbling' action of small-scale turbulent eddies (e.g. Corrsin $\&$ Kistler 1955) while others were based on the 'engulfing' motions of large-scale eddies and the 'elastic' dynamics of turbulence distorted by these eddies (Townsend 1966). Hypotheses about the jump in the large-scale vorticity were made in these models (e.g. Kovasznay, Kibens \& Blackweld 1970). With the aid of particle imaging velocimetry (PIV) systems and large computers running at mega- and tera-flops speed, the flow and scalar fields in these 'super-layers' can be measured and computed in sufficient detail to test the various hypotheses and theoretical models based on them (Westerweel et al. 2002, 2005; Bisset, Hunt \& Rogers 2002; Mathew \& Basu 2002).

The turbulence has special features in these thin layers which differ from those in the interior of most shear flows. In the interior region the length scale of the turbulent eddies $L_{x}$ is generally smaller than the distance $\Lambda$ over which the turbulence and 
mean velocity change. Typically $\Lambda$ is about $1 / 4$ of the shear layer thickness, $l$. By contrast adjacent to these thin 'super-layers' of thickness $l_{I}$ the turbulence is very inhomogeneous since the eddies controlling their dynamics are comparable to $l$ (where $l \gg l_{I}$ ). At the same time the layers are fluctuating and therefore have to be analysed in a coordinate frame moving with the interface (defined over a suitable length scale that is large compared to $l_{I}$ ). This invalidates the conventional statistical models based on assuming relations between mean gradients of turbulent fluxes and gradients of mean velocity/scalar (Durbin \& Petterson Reiff 2000; Hunt \& Savill 2002). An important consequence is that Reynolds-averaging methods and models are not valid near these layers because the relations between the ensemble mean and fluctuating components of the velocity field are quite different to those in less inhomogeneous regions of the flow.

Understanding the dynamics of these thin layers is also relevant for analysing the dynamics of coherent eddy motions both in homogeneous and inhomogeneous turbulence. At high Reynolds number such eddies tend to be defined by sharp interfaces separating highly turbulent and low-turbulence regions, where intense shear layers are formed. Similar discontinuities occur in scalar concentrations (Warhaft 2000). Within turbulent flows these layers appear as a form of interior intermittency (Vassilicos 2001). In the inhomogeneous regions between high- and low-intensity turbulence the small eddies in these intense shear layers strongly influence the speed at which the layers move outwards into the non-turbulent or low-turbulence regions of flow. This is the boundary entrainment velocity $E_{b}$ (Turner 1986).

For some flows, such as jets and plumes, where the mean velocity in the turbulence is much greater than in the non-turbulent region and $E_{b}$ is finite, and turbulent flow drives a mean velocity into the turbulent region - this is the relative entrainment velocity $E_{v}$. Estimates of the values of $E_{v}$ and $E_{b}$ in terms of other flow parameters are used for calculating many types of turbulent shear flows that occur in practice. However, there is no general theory or even empirical rule for determining these 'velocities' as circumstances change, for example when the level of turbulence inside or outside the region is suddenly changed (Bhat \& Narasimha 1996; Ching, Fernando \& Robles 1995; Hunt 1995) or when the layer is positioned between a cylindrical region of static fluid and a rotating annulus of highly turbulent flow, such as a hurricane (e.g. Bengston \& Lighthill 1982). Interfaces with inhomogeneous turbulent flows occur widely in geophysical flows, notably 'geostrophic' and gravity current fronts (Britter \& Simpson 1978).

The paper is structured as follows: in $\S 2$, we review the major results from linearized analysis near sharp interfaces bounding turbulent and non-turbulent regions. The kinematics and dynamics of interfacial layers bounding turbulent regions are described in $\S 3$, before applying these new results to swirling shear and boundary layers in $\S 4$. These results are put into a general context in $\S 5$.

\section{Turbulence near interfaces and thin vortical layers}

\subsection{Interfaces adjacent to layers with weak mean shear}

Consider an idealized analysis of two regions of fluctuating motion initiated at $t=0$, separated by a fluctuating interface located at $\hat{x}_{3}=0$, where $\hat{x}_{3}=x_{3}-x_{3 I}(t)$. The velocity field $\boldsymbol{u}=\left(u_{1}, u_{2}, u_{3}\right)$ is irrotational above the interface (figure 1) - we will use the superscripts $+/-$ to denote processes above/below the interface. For the shear-free flows (SFL) considered here the turbulence far below the interface is homogeneous, denoted by $\boldsymbol{u}^{(H)}$ and the mean velocity $\boldsymbol{U}=\left(U_{1}, U_{2}, U_{3}\right)$ is uniform. This homogeneous 


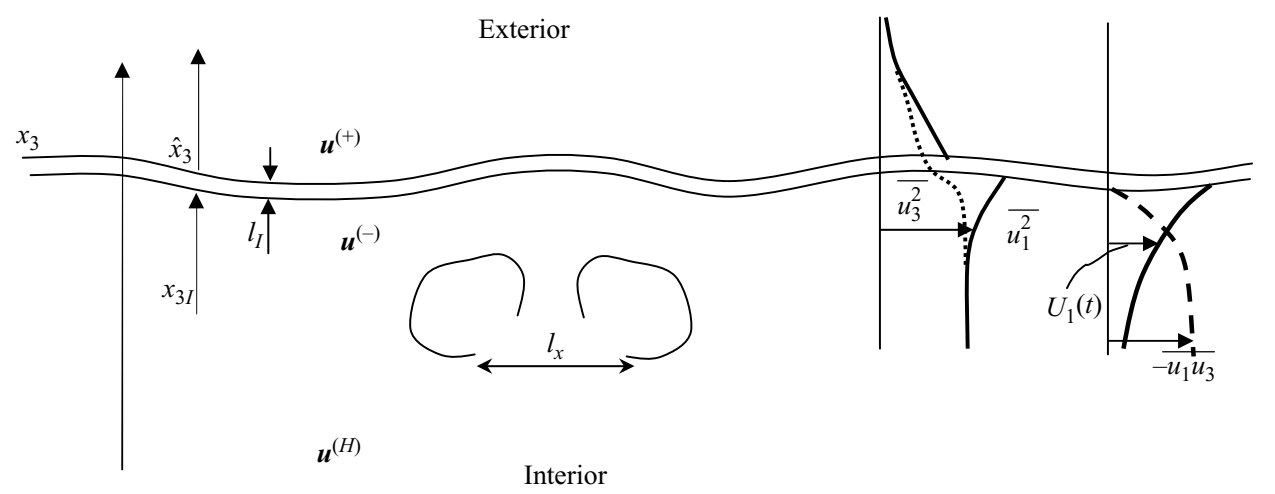

FIGURE 1. Showing schematically the inhomogeneous turbulence near the interface (at $x_{3}=x_{3 I}$, or $\hat{x}_{3}=0$ ) between a region of shear-free homogeneous turbulence and irrotational fluctuations. Note that if the turbulence $\boldsymbol{u}^{(H)}$ is anisotropic (e.g. with a Reynolds stress) the inhomogeneity leads to a Reynolds stress gradient and the growth of a mean shear layer $U_{1}(t)$.

turbulence is in a quasi-equilibrium state. The dissipation rate is $\epsilon$. The mean and fluctuating components of vorticity are $\left(\Omega_{1}, \Omega_{2}, \Omega_{3}\right)=\boldsymbol{\nabla} \times \boldsymbol{U}$ and $\boldsymbol{\omega}=\boldsymbol{\nabla} \times \boldsymbol{u}$. This idealized flow situation corresponds approximately to that in a wind tunnel where there is a grid of bars below $\hat{x}_{3}=0$ and a gauze above this level so that the net resistance to the flow is the same and the mean velocity uniform (Gartshore, Durbin \& Hunt 1983).

The main features of inhomogeneous turbulence near such interfaces can be studied by assuming that the adjustment of these fields is 'rapid', taking place on a time scale $T_{D}$, which is shorter than the Lagrangian time $T_{L}=L_{x} / u_{0}$ over which the large eddies evolve, where $L_{x}$ is the integral scale of the turbulence. Consequently, the broad structure of turbulent eddies near the interface can be estimated using approximate linear calculations. In shear-free flows, unlike sheared flows, the fluctuating vorticity of the turbulence is not affected over this time scale. Also the external field is simply irrotational, following Phillips (1955) and Carruthers \& Hunt (1986), so that

$$
\boldsymbol{u}^{(+)}=\nabla \phi^{(+)}, \quad \boldsymbol{u}^{(-)}=\nabla \phi^{(-)}+\boldsymbol{u}^{(H)},
$$

where $\left|\nabla \phi^{( \pm)}\right| \rightarrow 0$ as $\hat{x}_{3} / L_{x} \rightarrow \pm \infty$. To satisfy continuity,

$$
\nabla^{2} \phi^{( \pm)}=0 \text {. }
$$

The interfacial matching conditions for flows in which viscous effects are neglected for the largest scales are continuity of the velocity normal to the interface and pressure, $p$, across the fluctuating boundary between the interior and exterior regions. The condition reduces to $u_{3}$ and pressure, $p$ (or $\phi$ ), being continuous at $\hat{x}_{3}=0$. Because the equations and boundary conditions are linear, the effects of varying the spectrum can be analysed by generalizing the Fourier methods of Batchelor \& Proudman (1954).

The main results of relevance to the dynamics of these interfaces in unstratified flows are:

(i) Over a distance $L_{x}$, below the interface the mean squared value of the velocity fluctuations $\left(\overline{u_{1}^{\prime 2}}, \overline{u_{2}^{\prime 2}}\right)$ parallel to the interface increase by $25 \%$ above the homogeneous level, while the mean squared value of the velocity fluctuations $\left(\overline{u_{3}^{\prime 2}}\right)$ normal to the interface component decreases (by $50 \%$ ). Just above the interface, $\overline{u_{1}^{\prime 2}}, \overline{u_{2}^{\prime 2}}$ are reduced 
to $25 \%$, showing that there is a discontinuity in eddy motions parallel to the interface caused by the vortex lines normal to the interface turbulence forming vortex sheets at the interface.

(ii) As Phillips (1955) showed, and many experiments have since confirmed, the variances of all velocity components are determined by the largest eddy scales and decay with normal distance in the external layer approximately in proportion to $\sqrt{\overline{u_{i}^{\prime 2}}} \sim A \hat{x}_{3}^{-4}$, for $\hat{x}_{3} \gg L_{x}$. This decay is not sensitive to the form of the lowwavenumber energy spectrum, provided $E(k) \sim C k^{N}$, where $N$ is an integer greater than or equal to 4 and $C \sim u_{0}^{2} L_{x}^{(N+1)}$ (see Davidson 2004). The linear analysis of (2.1), (2.2) shows that $A \sim \int E(k) / k^{4} \mathrm{~d} k \sim C / L_{x}^{(3-N)} \sim u_{0}^{2} L_{x}^{4}$.

(iii) The spectrum of velocity fluctuations changes sharply across the interface. Below the interface, there is a fully developed spectrum with a Kolmogorov inertial range extending to the smallest scales of order $\left(v^{3} / \epsilon\right)^{1 / 4}$, where $\epsilon$ is the dissipation rate and $v$ the kinematic viscosity. Above the interface, the energy spectrum is concentrated at low wavenumbers so that the length scale of the most energetic straining motions is of order $L_{x}$. However, since there is a 'jump' in the parallel components of velocity across the interface, a fluctuating viscous layer is a generated. Because the straining motions distorting this layer are of a relatively large scale (of order $\left.L_{x}\right)$, the layer thickness is comparable to the Taylor microscale, i.e. $\left(L_{x} / u_{0}\right)^{1 / 2}$. No detailed measurements are yet available.

The dynamics near the interface are significantly changed if the interior turbulence $\boldsymbol{u}^{(H)}$ is anisotropic. The distortion of such turbulence near a rigid plate has been analysed and measured by Nagata et al. (2005) and Wong (1985), and can be extended to account for the distortion of anisotropic turbulence near the interface. When turbulence impinges onto an interface or a rigid boundary, there are gradients in the normal Reynolds stress (i.e. $\partial \tau_{i j} / \partial x_{j}$, where $\tau_{i j}=-\left\langle u_{i} u_{j}\right\rangle$ ). For isotropic turbulence these do not generate a mean velocity field. However, if the curl of the Reynolds stress gradients is non-zero, this amplifies the mean vorticity $\Omega_{i}$, i.e. $\partial \Omega_{i} / \partial t=\epsilon_{i j k} \partial \tau_{k m} / \partial x_{j} \partial x_{m}$. In this case, for anisotropic turbulence impinging onto an interface, $\Omega_{2}=\partial U_{1} / \partial x_{3}$ and thence $U_{1}$ gradually increase below the interface, with a discontinuity in $U_{1}$ at $\hat{x}_{3}=0$. This is illustrated by a model calculation, in which there is a mean Reynolds stress (normalized on the density) $\tau_{13}=-\left\langle u_{1} u_{3}\right\rangle$ below the interface. Consider a two-dimensional single Fourier mode of the homogeneous turbulence below the interface:

$$
u_{1}^{(H)}=u_{0} \sin \psi \cos \left(-k_{1} x_{1}+k_{3} \hat{x}_{3}\right), \quad u_{3}^{(H)}=u_{0} \cos \psi \cos \left(-k_{1} x_{1}+k_{3} \hat{x}_{3}\right),
$$

where $k_{1}=\tilde{k} \cos \psi, k_{3}=\tilde{k} \sin \psi$ are the wavenumbers in the $x_{1^{-}}$, $\hat{x}_{3}$-coordinates. Then applying the same equations and forms of solution as in (2.1) and (2.2) it follows that $\tau_{13}\left(\hat{x}_{3}\right)=-\frac{1}{4} u_{0}^{2} \sin 2 \psi-\frac{1}{2} u_{0}^{2} \cos \psi \sin \left(k_{3} \hat{x}_{3}-\psi\right) \mathrm{e}^{\left|k_{1}\right| \hat{x}_{3}}$. Note that $\tau_{13}$ decreases over a distance $L_{x}$ from its homogeneous value to zero at and above the interface. For the simple model perturbation of (2.3), the mean profile becomes

$$
U_{1}=-\frac{1}{4} u_{0}^{2} t \tilde{k} \sin 2 \psi \cos \left(k_{3} \hat{x}_{3}-\psi\right) \mathrm{e}^{\left|k_{1}\right| \hat{x}_{3}}, \quad \hat{x}_{3}<0, \quad t>0 .
$$

Note that this mechanism of generating a mean flow is similar to that found when waves approach a beach or a critical layer at an angle. Despite the neglect of significant non-linear effects, these linear rapid distortion analyses of inhomogeneous turbulent flows are essentially local linearizations of complex flows for which physical and theoretical justification have been given by Hunt \& Carruthers (1990) and 


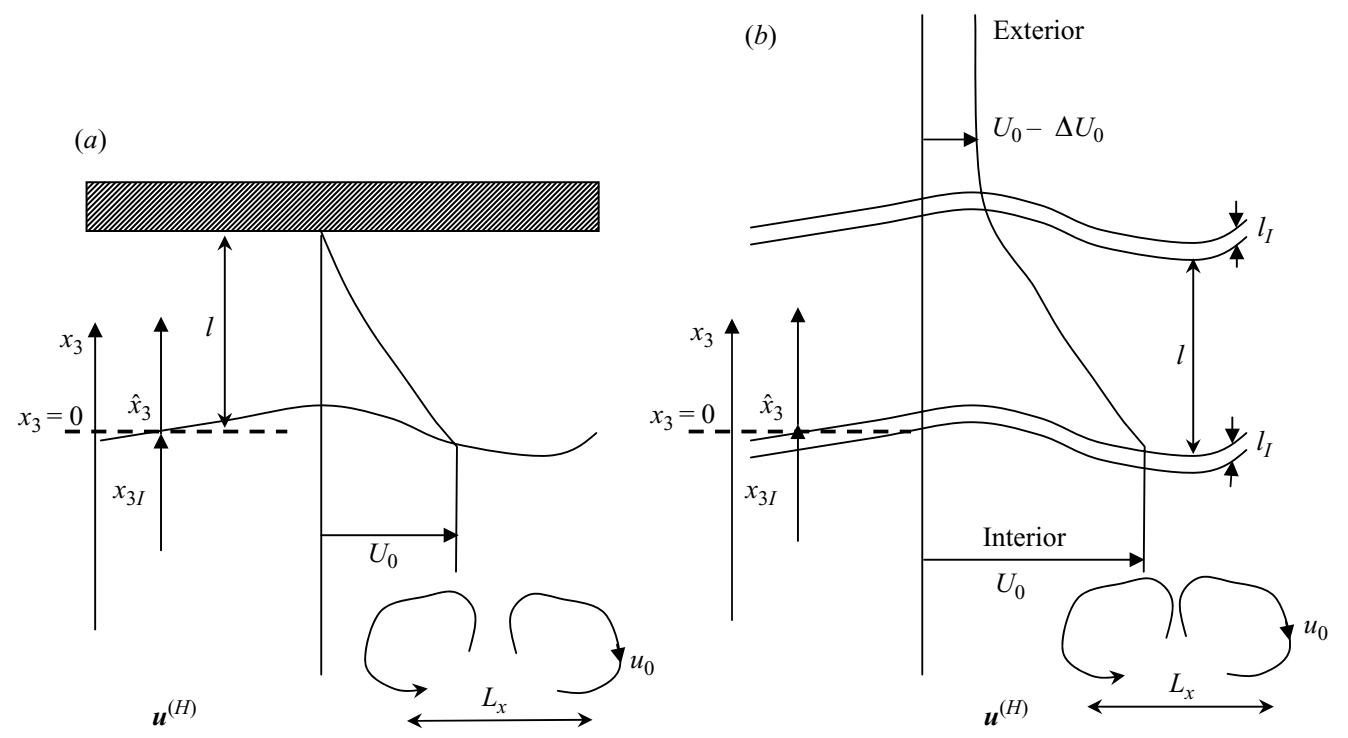

FIGURE 2. (a) Boundary-shear layers (BSL) and (b) free-shear layers (FSL) where interior turbulence induces rotational fluctuations in the shear layer of thickness $l$. In case $(b)$, irrotational fluctuations occur in the exterior region.

Magnaudet (2003). Similar effects occur whether these fluctuations are space filling (e.g. Fourier waves) or compact (e.g. vortices).

\subsection{Turbulence near layers with a strong mean shear}

Now consider a similar idealized flow involving interactions between large-scale turbulence and adjacent, external, regions where initially there is no turbulence. Two different flow situations are considered, broadly categorized as either boundary-shear layers (BSL - figure $2 a$ ) or free-shear layers (FSL - figure $2 b$ ), depending on whether the shear layers are bounded by a rigid boundary or by an external layer in which the mean velocity is uniform (see figure $2 b$ ). Irrotational velocity fluctuations $\left(\boldsymbol{u}^{(+)}\right)$ generated in the shear layer are present in this external region (Hunt \& Durbin 1999). In both cases the mean velocity profile in the shear layer is unstable to small disturbances which become non-linear and develop into turbulent eddies. These grow in scale until they are as large as the thickness of the shear layer. Through entrainment (discussed below in $\S 3$ ) the shear layer thickness $l$ grows with time. There are sharp interfaces with thickness $l_{I} \ll l$ separating the shear layer from the external and lower turbulent regions. When there are weak fluctuations in the lower layer with lengthscales greater than $l$, they interact linearly with the layer. If they are large enough (see below) they may affect fluctuations throughout the layer. In the case of FSL, they generate fluctuations on the other side of the layer where $\hat{x}_{3}>l$. Linear dynamics of wave-like disturbances and vortical eddies determine the broad spatial structure of the fluctuations both near interfaces $(\S 2.1)$ and where the turbulence is homogeneous in thick shear layers (e.g. Townsend 1976).

An inviscid analysis of these idealized flows (approximately valid for large $R e=$ $u_{0} L_{x} / v \gg 1$ ) combines a perturbation analysis for interactions across interfaces (as in § 2.1) and a linear rapid distortion approach for the development of fluctuations in shear flows. The analysis for the energy-containing eddies is approximately valid for distortion times shorter than the Lagrangian time $T_{L}=L_{x} / u_{0}$. These studies show 
how the amplitude of externally imposed velocity fluctuations generally decreases in the turbulent region near the interface at $\hat{x}_{3}=0$. But above the interface, their structure depends on how the fluctuations in the shear layer are initiated.

In the turbulent layer $\left(\hat{x}_{3}<0\right)$ (as in $\left.\S 2.1\right)$, the flow consists of homogeneous turbulence and irrotational fluctutations, i.e.

$$
\boldsymbol{u}^{(-)}=\boldsymbol{u}^{(H)}+\nabla \phi^{(-)} .
$$

We consider the case where the shear layer is thick enough that $l \gg L_{x}$. Linearized momentum equations are valid in the shear layer $0<\hat{x}_{3}<l$, for $t \ll L_{x} / u_{0}$, namely

$$
\left(\frac{\partial}{\partial t}+\boldsymbol{U} \cdot \nabla\right) \boldsymbol{u}+\boldsymbol{u} \cdot \nabla \boldsymbol{U}=-\nabla p,
$$

where $p$ is the pressure normalized by density. Note that for uniform shear this reduces, for the vertical component, to

$$
\left(\frac{\partial}{\partial t}+\boldsymbol{U} \cdot \nabla\right) \nabla^{2} u_{3}=0 .
$$

For FSL in the upper layer,

$$
\boldsymbol{u}^{(+)}=\nabla \phi^{(+)} .
$$

Given $\boldsymbol{u}^{(H)}$ in the turbulent layer, the velocity fluctuations in the other layers are determined by the above equations and initial, matching and boundary conditions. At $\hat{x}_{3}=0, u_{3}$ and $p$ are continuous. For BSL, an additional kinematic constraint, $u_{3}=0$ at $\hat{x}_{3}=l$, is applied.

\subsubsection{Boundary-shear layers ( $B S L$ )}

In the first type of boundary-shear layer (BSL), velocity fluctuations in the upper layer $\left(\hat{x}_{3}>0\right)$ are initiated in a time-dependent flow at $t=0$. Equivalently they could be generated in a spatially developing flow for $x_{1}>0$ by a turbulent flow passing below a rigid plate extending from $-\infty<x_{1}<0$ (where the plate produces a negligible wake). Initially the velocity fluctuations above and below the interface are described by the shear-free analysis of $\S 2.1$. But as time $t$ ( or $x_{1}$ ) increases, the mean shear in the layer amplifies the horizontal fluctuations $u_{1}, u_{2}$ and the Reynolds stress $-\left\langle u_{1} u_{3}\right\rangle$. By contrast (from (2.7)), the vertical fluctuations do not decay, as they do (in linear theory) when homogeneous rotational disturbances perturb a uniform shear layer (e.g. Townsend 1976). This is a simple demonstration of how inhomogeneous turbulence can have features that differ qualitatively from those in uniform shear flows. These interfacial processes were broadly confirmed by the wind tunnel experiment of Gartshore et al. (1983).

In the second type of BSL flow, the turbulence is externally forced with no velocity fluctuations in the shear layer at $t=0$. For example this might be a laminar boundary layer on a plate (at $\hat{x}_{3}=l$ ) where external turbulence $\boldsymbol{u}^{(H)}$ is generated in the space below $\hat{x}_{3}<0$, or where vortices propagate towards the boundary layer (see figure $2 a$ ). Linear theory (e.g. Lighthill 1957) and computations show that fluctuations $u_{l}$ on a scale $l$ are directly related to the pressure fluctuations in the turbulent layer $p^{(-)}$; approximately $u_{l} \sim p^{(-)} / U_{1}\left(x_{3}\right)$. If the eddies or waves travel at the uniform external flow speed where $U_{1}=U_{0}$, the linear inertial terms induce a fluctuating pressure of order $u_{0}\left(U_{0}-c\right)$ (Grosch \& Salwen 1978; Craik 1991). Weak nonlinear inertial terms exist where $p^{(-)} \sim u_{0}^{2}$. Consequently the fluctuations in the shear layer $\left(\hat{x}_{3}>0\right)$ are very weak, i.e. $u_{l} \sim u_{0}^{2} / U_{1}\left(x_{3}\right)$ (Jacobs \& Durbin 1998). A physically analogous 
mechanism occurs when a vortex approaches such a layer: the vortex distorts the vorticity of the layer and blocks the motion of the vortex towards the layer (Hunt $\&$ Durbin 1999). This means that the interface is effectively acting as a barrier, which therefore makes the velocity fluctuations anisotropic as eddies and vortices approach the interface; the vertical velocity $u_{3}$ decreases while the horizontal velocity components are increased. Therefore a fluctuating vortex sheet is created which as a result of nonlinear effects soon becomes unstable leading to spots of turbulence propagating into the shear layer (Wu et al. 1999). This phenomenon, which accelerates the transition to turbulence of laminar boundary layers, has been used to improve the design of turbomachinery (Hodson \& Howell 2005).

\subsubsection{Free-shear layers (FSL)}

Now consider the interaction between the external turbulence (with r.m.s. velocity $u_{0}$ ) and a free-shear layer (figure $2 b$ ) where the mean velocity $U_{0}-\Delta U_{0}$, in the upper turbulent free-shear layer differs from that in the lower turbulent layer by $\Delta U_{0}$. The magnitude of the mean velocity difference is much greater than the r.m.s. turbulence in the lower layer, i.e. $\Delta U_{0} \gg u_{0}$.

In the idealized calculation we now discuss it is assumed that the shear layer is much thinner than the turbulent length scale (i.e. $l \ll L_{x}$ ), and behaves like a perturbed vortex sheet. The mean vorticity $\Omega_{2}$ in the layer is much greater than the strain rate of the energy-containing eddies, i.e. $\left|\Omega_{2}\right| \sim \Delta U_{0} / l \gg u_{0} / L_{x}$. Nevertheless the thickness and displacement of the layer fluctuates with $x_{1}, x_{2}, t$. The layer is of course exponentially unstable to small disturbances, causing alternating thickening and thinning of the vortex sheet. Where the former occurs characteristic vortex structures develop (Batchelor 1967) which determine the growth of the mean thickness of the layer. The thickening/thinning mechanism is also relevant to understanding how the shear layer responds to large-scale external fluctuations (Hunt \& Durbin 1999). To illustrate these processes, consider a single Fourier mode of the turbulence travelling at speed $c$ parallel to the mean flow, e.g.

$$
u_{3}^{(H)}=u_{0} \mathrm{e}^{\mathrm{i}\left(k_{1}\left(x_{1}-c t\right)+k_{3} \hat{x}_{3}\right)}, \quad \hat{x}_{3}<0 .
$$

Because the layer is thin (as with the analysis of the Kelvin-Helmholtz instability) the vertical velocity $u_{3}$ is constant within the layer while $u_{1}, u_{2}$ vary linearly across it. The irrotational flow field (2.8) in the upper layer is determined by ensuring continuity of $u_{3}$ and pressure $p$. Solving the coupled equations shows that travelling eddies in the turbulent flow produce irrotational fluctuating velocities in the upper region that travel in phase and do not grow in amplitude. If the external eddies are large enough this quasi-steady interaction is independent of the growth of fluctuations within the free-shear. A linear analysis for two-dimensional fluctuations shows that the ratio of the standard deviations of the velocity fluctuations in the upper layer to that in the turbulent flow far from the interface is

$$
\frac{\sqrt{\overline{u_{3}^{(+)^{2}}}}}{\sqrt{\overline{u_{3}^{(H)^{2}}}}}\left(\hat{x}_{3} \sim l\right)=\frac{b}{2}\left|\frac{\Delta U_{0}^{2}-4 c_{r}^{2}}{\Delta U_{0}^{2}+4 c_{r}^{2}}\right|
$$

(Hunt \& Durbin 1999), where $c_{r}=c-\left(U_{0}-\Delta U_{0} / 2\right)$ is the wave speed relative to the mean speed in the interface. The ratio (2.10) is zero for $c=U_{0}$, i.e. when the vortex sheet shelters the perturbations. If it is non-zero it only depends weakly 
on the anisotropy of the turbulence, as shown by the form of the coefficient $b=$ $\left.\overline{\left(u_{1}^{(H)^{2}}\right.}+\overline{u_{3}^{(H)^{2}}}\right) / 2 u_{0}^{2}$.

This quasi-steady blocking mechanism can also be studied by considering the thickening and thinning of the vortex sheet as a vortex moves. Most flow disturbances have an impulse (or in Charles Dickens' (1865) words, 'impulsion', the literary concept preceding Kirchhoff's (1869) analysis by 4 years!). Therefore an equivalent impulse must be generated by the deformation of the sheet, according to the general formula

$$
\boldsymbol{I}=1 /(N-1) \int \boldsymbol{x} \times \boldsymbol{\omega} \mathrm{d} \boldsymbol{x}
$$

where the integral is taken over the volume occupied by the sheet and $N=2,3$ depending whether the sheet is one- or two-dimensional (e.g. Batchelor 1967). This analysis demonstrates how the greater circulation associated with the thicker regions of the sheet can induce a vertical velocity component opposite to that of the impinging vortex, so that depending on the speed of the vortex parallel to the sheet, (i.e. the value of $c / U_{0}$ ) the sheet may or may not block the vertical velocities associated with the vortex. As in the case of BSL, the blocking of the vertical fluctuations leads to an amplification of the horizontal velocity components at the interface where there is a weak mean shear flow $U\left(x_{3}\right)$ below the vortex sheet, such that $\left|\mathrm{d} U / \mathrm{d} x_{3}\right| \lesssim U_{0} / L_{x}$, it can be shown (Hunt et al. 2006) that the mean shear is amplified by vortex stretching until it reaches a steady state. This depends on the shape of the impinging eddies. For typical shapes it can be shown that $\mathrm{d} U\left(x_{3}\right) / \mathrm{d} x_{3} \propto U_{0} /\left|x_{3}\right|$.

This shear sheltering/blocking mechanism also affects the distribution of scalars in these inhomogeneous flows. For example, if there is a vertical gradient of mean concentration such that $C\left(x_{3}\right)$ increases away from the interface, then as the vortical eddy approaches the interface and as it displaces fluid it transports the scalar with it. But the scalar perturbation is confined to the region around the eddy whereas the irrotational fluctuations induced around the vortex can amplify the vorticity in the shear layer (e.g. Wu et al. 1999). In this case, momentum can be transported more effectively than a scalar. This is an exceptional phenomenon in unstratified turbulent flows (see Townsend 1976), but has been observed in the measurements of Smedman et al. (2006) for a boundary layer flow where eddies impinge onto a rigid boundary.

\section{Kinematics and dynamics of interfacial layers}

Observations, measurements and detailed numerical simulations of turbulent shear flows (e.g. Westerweel et al. 2002, 2005; Mathew \& Basu 2002; Bisset et al. 2002) show the complex highly distorted interface bounding the edge of turbulent regions (see figure 3). The main difference between the idealized flat interface of $\S 2$ and observations, is that periodically the interface forms cusps that point inwards towards the turbulent region $\left(\hat{x}_{3}<0\right)$ and smooth bulges in the opposite direction. The local processes associated with these inward cusps or outward bulges are significant for the overall flow because they affect the transport of material (and scalars) into the turbulent region from outside $\left(\hat{x}_{3}>0\right)$. To study these processes, the interfacial processes need to be examined in a coordinate system moving with the interface, as we go on to discuss.

\subsection{Kinematics and jump conditions at interfacial shear layers}

Many features of the overall dynamics of shear layers can be explained in terms of the mechanisms in the interfacial layers. This is not the usual methodology, although 
(a)
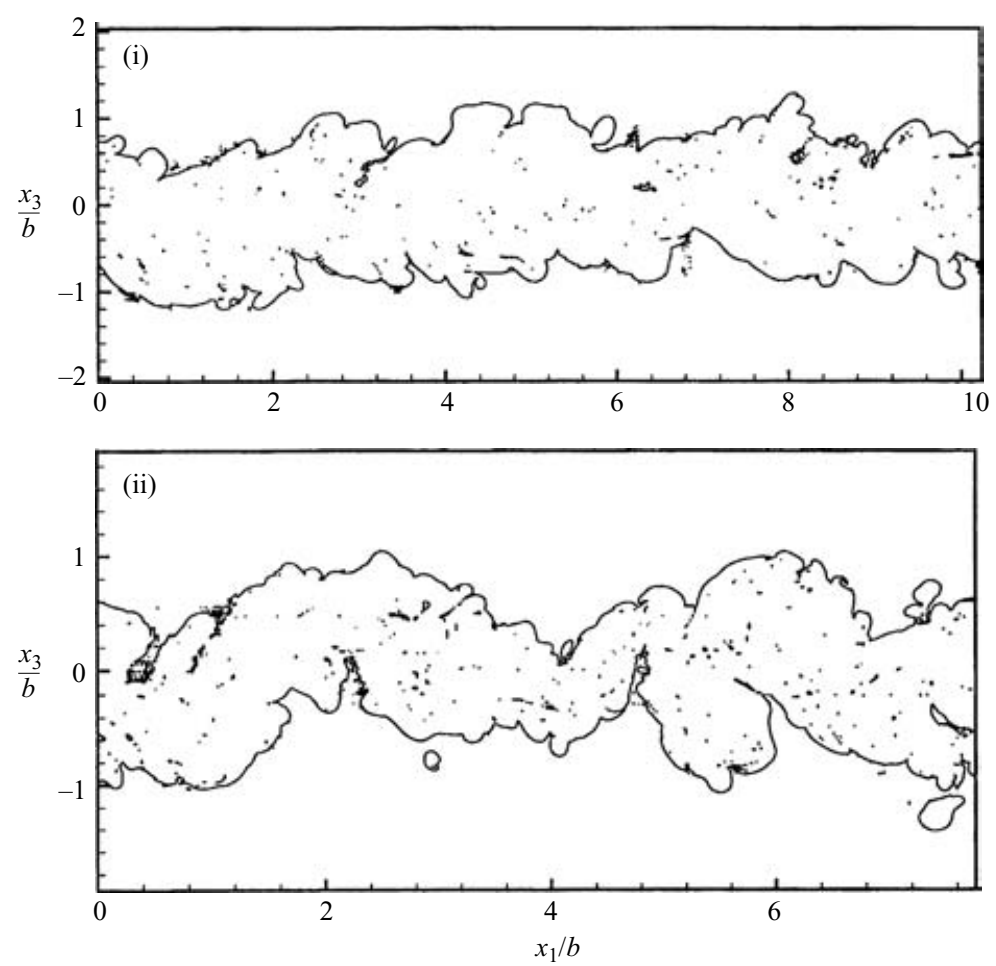

(b)

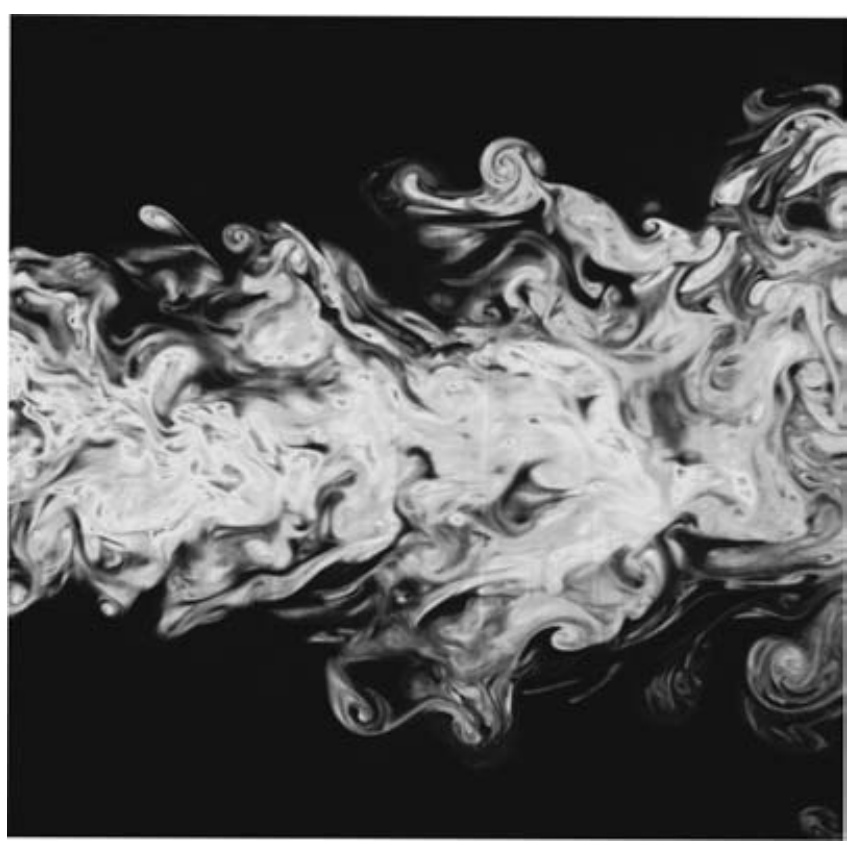

FIGURE 3. Numerical simulations and experimental observations of the interface bounding a wake and a jet. (a) Edge of (i) an unforced axisymmetric wake and (ii) a weakly forced wake; $b$ is the mean radius of the wake. The contour is a threshold of vorticity (from Bisset et al. 2002). (b) Experimental observations using LIF of the distribution of a passive scalar in a three-dimensional turbulent jet (from Westerweel et al. 2005). The jet is flowing from the leftto the right-hand side of the figure. 
(a)

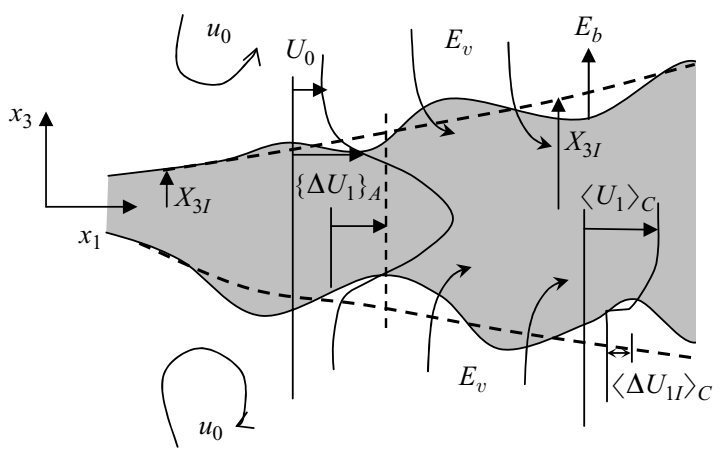

(b)

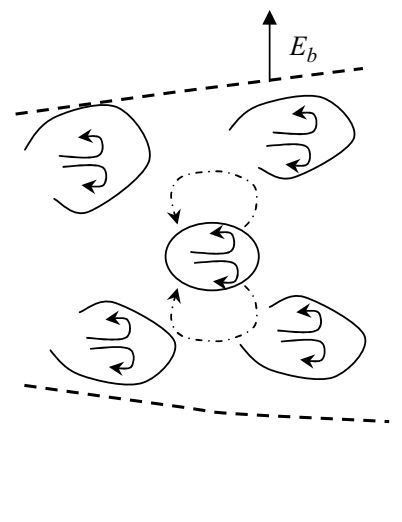

FIGURE 4. Schematic diagram of two stages in the development of a turbulent jet (or plume) moving in an external stream with mean velocity $U_{0}$ and r.m.s. turbulence $u_{0}$. $(a)$ In the initial stage, the mean excess velocity $\left\{\Delta U_{1}\right\}_{A}$ and the turbulence $\Delta \overline{u_{1}^{2}}$ in the jet are greater than $u_{0}$. Note the difference between the inward entrainment velocity $E_{v}$ and the outward entrainment velocity $E_{b}$. (b) In the far downstream stage, the jet has broken into eddies, but the momentum flux is unaffected. The entrainment velocity is $E_{v}=0$, while $E_{b} \neq 0$, where - - - denotes the edge of the jet.

it was the essential concept in G. I. Taylor's (1958) theoretical model for turbulent jets where he focused on the mean normal 'entrainment velocity' $E_{v}=-\left\langle u_{3}\right\rangle$, where $\langle\cdot\rangle$ is the ensemble mean which is directed into the turbulent region (see figure 4). For a circular jet propagating in the $x_{1}$-direction, mass conservation requires

$$
E_{v}=\frac{1}{2 \pi R} \frac{\mathrm{d} Q}{\mathrm{~d} x_{1}}
$$

where the volume flux $Q$ is defined by

$$
Q=\Delta U_{0} \pi R^{2},
$$

and where $R$ is the mean jet radius and $\Delta U_{0}$ is the difference between the mean velocity in the jet and the external flow. Morton, Taylor \& Turner (1956) introduced the assumption for turbulent jets and plumes that the ratio

$$
\alpha_{v}=\frac{E_{v}}{\Delta U_{0}}
$$

is constant. However (cf. Hunt 1995; Agrawal \& Prasad 2004; Bhat \& Narasimha 1996), this coefficient is not constant when jets are perturbed by body forces or external turbulence.

A critical measure of the interfacial dynamics is the average speed $E_{b}$ of the boundary interface between the turbulent and non-turbulent flows:

$$
E_{b}=\left\langle\frac{\mathrm{d} X_{3 I}}{\mathrm{~d} t}\right\rangle
$$

where the derivative is a Lagrangian following the random motion of fluid particles in the interface. Here $X_{3 I}$ is the instantaneous position defined as the value of $x_{3}$ where relevant properties of the flow or a scalar field are invariant. For example, the interface position can be defined as where the difference between the average value of the variance just outside the interface $\left\langle u_{i}^{2}\right\rangle_{I}$ and the r.m.s external turbulence $\left(\overline{u_{i}^{2}}\right)_{0}$, 
normalized on the square of the difference $\Delta u_{0}^{2}$ between the r.m.s levels in the two regions, is small, say $\tilde{\sigma}_{u}^{2}=0.1$, e.g.

$$
\frac{\left\langle u_{i}^{2}\right\rangle_{I}-\left(\overline{u_{i}^{2}}\right)_{0}}{\Delta u_{0}^{2}}\left(x_{3}=X_{3 I} ; x_{1}, t\right)=\tilde{\sigma}_{u}^{2} .
$$

The interface position may also be defined in terms of conditional mean velocity or scalar differences, e.g. see figure 3(a) (Bissett et al. 2002).

In free-shear layers (FSL), there is a jump $\left\langle\Delta U_{1 I}\right\rangle_{C}$ in the conditional mean velocity $\left\langle U_{1 I}\right\rangle_{C}$ across the fluctuating interface, at $x_{3}=X_{3 I}$, and a mean shear flow $\left\langle U_{s}\right\rangle_{C}$ below the interface. This is equivalent to a delta-function in the vorticity field, i.e. $\left\langle\Delta \Omega_{2}\right\rangle_{C}=\left\langle\Delta U_{1 I}\right\rangle_{C} \delta\left(x_{3}-X_{3 I}\right)$. The total conditional velocity is

$$
\left\langle U_{1}\right\rangle_{C}=U_{0}-\left\langle\Delta U_{1 I}\right\rangle_{C}+\left\langle U_{s}\right\rangle_{C}, \quad x_{3}<\left\langle X_{3 I}\right\rangle, \quad\left\langle U_{1}\right\rangle_{C}=U_{0}, \quad x_{3}>\left\langle X_{3 I}\right\rangle .
$$

Numerical simulations (Bisset et al. 2002) and experiments (Westerweel et al. 2002, 2005) show that in the limit of very high Reynolds numbers there should be a jump in the conditionally averaged Reynolds stress $\left\langle\Delta \tau_{I}\right\rangle_{C}$ across the interface which drives the mean acceleration of the local flow associated with the interface moving outwards (see figure $4 a$ ). For a local dynamical balance in a steady frame moving with the interface,

$$
E_{b}\left\langle\Delta U_{I}\right\rangle_{C}=\left\langle\Delta \tau_{I}\right\rangle_{C}
$$

(see Westerweel et al. 2005, and $\$ 2.2$ ). In the interior of the shear layer, where there is locally a homogeneous turbulent shear flow, the magnitude of $\left\langle\Delta \tau_{I}\right\rangle_{C}$ is determined by the mean shear and the local turbulent eddy viscosity $\nu_{T}$ (Townsend 1976),

$$
\langle\Delta \tau\rangle_{C} \approx v_{T}\left\langle\frac{\partial U_{s}}{\partial \hat{x}_{3}}\right\rangle_{C},
$$

where $v_{T}=0$ for $\left.x_{3}\right\rangle\left\langle X_{3 I}\right\rangle$. By averaging the relation between the mean shear stress (in fixed coordinates) $\Delta \tau\left(x_{3}\right)$ and mean shear $\partial U_{1} / \partial x_{3}$ over all the fluctuating positions of the interface, the usual eddy viscosity at fixed values of $x_{3}$ can be derived (Westerweel et al. 2005), i.e.

$$
\left\langle v_{T}\right\rangle \approx \Delta \tau / \frac{\partial U_{1}}{\partial x_{3}} .
$$

Interestingly this ratio is approximately constant, as assumed by Prandtl (1925) and in many basic engineering models. But (3.9) is inconsistent with Reynolds-averaged stress models (see Hunt et al. 2001) where it is assumed that $\left\langle v_{T}\right\rangle$ tends to a very small value in the exterior region (but usually larger than $v$ ).

When there are strong local body forces (or obstructions), the jump conditions are quite different and can determine the whole flow structure. In strongly rotating or stratified flows the eddy diffusivity and the boundary entrainment velocity can be greatly reduced, i.e. $v_{T} / u_{0} l \ll 1$ and $E_{b} / u_{0} \ll 1$ (Turner 1973). Then the jump in shear stress across the interface is negligible, even though there is a jump in the mean velocity, $\left\langle\Delta U_{1 I}\right\rangle_{C}$. This requires that the thickness of the layer has to be large enough for the turbulence generated to be sufficiently distorted by body forces and $\left\langle\Delta \tau_{I}\right\rangle_{C} / u_{0}^{2} \ll 1$. A different mechanism operates in shear flows in cases where mean and fluctuating body forces extend into the turbulent layer by a distance $l_{B I}$, the entrainment velocity $\left(E_{b} / u_{0}\right)$ is zero, but the dynamical balance leads to a jump in the shear stress $\left\langle\Delta \tau_{I}\right\rangle_{C}=-\left\langle f_{I}\right\rangle_{C} l_{B I}$ (Coceal \& Belcher 2004; Grachev \& Hunt 2006). 


\subsection{Dynamics and entrainment at free surfaces in unidirectional shear layers}

The overall dynamics for unidirectional shear layers with no external pressure gradients is first of all determined by the growth of the mean momentum flux $(M)$

$$
\frac{\mathrm{d} M}{\mathrm{~d} x_{1}}=F_{1}=\int_{A} f_{1} \mathrm{~d} A
$$

and the volume flux $Q$ (see (3.1)). Here the cross-sectional area $A$ spans the jet/plume/boundary layer and $F_{1}$ is the integral of the mean body force $f_{1}$ over the jet acting in the flow direction.

For a statistically steady free round jet placed in an external flow with uniform velocity $U_{0}$, no pressure gradient and turbulence $\overline{u_{0 i}^{2}}$, the excess momentum flux is determined by the mean velocity and the excess turbulence, namely

$$
M=\int_{A}\left(\Delta U_{1}^{2}+\Delta \overline{u_{1}^{2}}\right) \mathrm{d} A=A \mu\left\{\Delta U_{1}\right\}_{A}^{2}\left(1+\lambda^{2}\right) \sim A\left\{\Delta U_{1}\right\}_{A}^{2}\left(1+\lambda^{2}\right),
$$

where $\left\{\Delta U_{1}\right\}_{A}=(1 / A) \int_{A} \Delta U_{1} \mathrm{~d} A, \Delta U_{1}=U_{1}-U_{0}, \Delta \overline{u_{1}^{2}}=\overline{u_{1}^{2}}-\overline{u_{01}^{2}}$ (overbar denotes an ensemble average at a fixed location), $\lambda$ denotes the average turbulence intensity defined by $\lambda^{2}=\left(\int_{A} \Delta \overline{u_{1}^{2}} \mathrm{~d} A\right) /\left(\int_{A} \Delta U_{1}^{2} \mathrm{~d} A\right) \sim \overline{u_{1}^{2}} /\left\{\Delta U_{1}\right\}_{A}^{2}$, and the $O(1)$ profile parameter $\mu=\left(\int_{A} \Delta U_{1}^{2} \mathrm{~d} A\right) / A\left\{\Delta U_{1}\right\}_{A}^{2}$. The physical significance of the contribution to the momentum flux produced by turbulence is often overlooked.

$M, Q$ and the external entrainment velocity $E_{v}$ are now related to the mean radius $R\left(x_{1}\right)$ and the boundary entrainment velocity $E_{b}$ (without necessarily assuming selfsimilarity profiles, e.g. Townsend 1976). By its definition (3.1), $E_{b}=\left\{\Delta U_{1}\right\}_{A} \mathrm{~d} R / \mathrm{d} x_{1}$, for a jet. From the physics of the interface $E_{b} \propto\left\{\Delta U_{1}\right\}_{A}$ so that $\mathrm{d} R / \mathrm{d} x_{1} \approx \alpha_{b}$, where $\alpha_{b} \approx 0.1$ is constant (Turner 1973). The external entrainment velocity (which is directed inwards) is calculated from continuity (see (3.1)) and is usually expressed by a coefficient $\alpha_{v j}$ in terms of the difference in the mean velocity inside and outside the shear layer, e.g. for a circular jet $E_{v}=\alpha_{v j}\left\{\Delta U_{1}\right\}_{A}, A=\pi R^{2}$, where

$$
\alpha_{v j}=\frac{1}{2 \pi R\left\{\Delta U_{1}\right\}_{A}} \frac{\mathrm{d}}{\mathrm{d} x_{1}}\left(A\left\{\Delta U_{1}\right\}_{A}\right) .
$$

By considering the momentum flux in (3.11), $M=A\left\{\Delta U_{1}\right\}_{A}^{2}\left(1+\lambda^{2}\right)$ is constant. Then from (3.12),

$$
\alpha_{v j}=\frac{\sqrt{1+\lambda^{2}}}{2} \frac{\mathrm{d}}{\mathrm{d} x_{1}}\left(\frac{\alpha_{b} x_{1}}{\sqrt{1+\lambda^{2}}}\right)
$$

Therefore if $\lambda^{2}$ is constant, as in a round jet with no external turbulence, $\alpha_{v j}=\alpha_{b} / 2$.

In the presence of external turbulence $\lambda^{2}$ is constant near the source of a jet where the level of turbulence exceeds the external level. But further downstream $\lambda^{2}$ increases beyond a transition point $\left(x_{1}^{*}\right.$, say) when the intensity of jet turbulence decreases to a level comparable to the external turbulence (Ching et al. 1995). For example, if

$$
\lambda^{2}=\lambda^{* 2}\left(1+\left(x_{1}-x_{1}^{*}\right) H\left(x_{1}-x_{1}^{*}\right) / R^{*}\right),
$$

where $H(\cdot)$ is the Heaviside-step function, then for $x_{1}>x_{1}^{*}$,

$$
\alpha_{v j}=\frac{\alpha_{b}}{2}\left[1-\frac{\lambda^{* 2} x_{1} / R^{*}}{2\left[1+\lambda^{* 2}\left(1+\left(x_{1}-x_{1}^{*}\right) / R^{*}\right)\right]}\right] .
$$


Circular jet unperturbed

Circular jet perturbed

Wake

Boundary layer

Swirling shear layer

Wall perturbed boundary layer

$\begin{aligned} E_{b} /\left\{\Delta U_{1}\right\}_{A} & \operatorname{Sign}\left(E_{v}\right) \\ \sim 1 & >0 \\ \sim 1 & >0 \\ \sim 1 & >0 \\ \sim 1 & >0 \\ \ll 1 & - \\ \sim 1 & -\end{aligned}$

$$
\left|E_{v} / E_{b}\right|
$$

$$
\sim 1 / 2
$$$$
<1 / 2
$$$$
\ll 1\left(\sim\left\{\Delta U_{1}\right\}_{A} / U_{0}\right)
$$$$
\ll 1\left(\sim\left\{\Delta U_{1}\right\}_{A} / U_{0}\right)
$$

$-$

TABLE 1. Entrainment velocities, $E_{b}, E_{v}$, for different types of shear layers.

Thus $\alpha_{v j} / \alpha_{b}$ has a step decrease at $x_{1}=x_{1}^{*}$ and then asymptotes to a value of $1 / 4$ when $x_{1}^{*} / R^{*} \gg 1$. This result demonstrates why in jets and plumes in the presence of free-stream turbulence, the ratios $\alpha_{v j} / \alpha_{b}$ and consequently $E_{v} / E_{b}$ or $E_{v} /\left\{\Delta U_{1}\right\}_{A}$ are not constant and can decrease quite suddenly when extra turbulence is introduced through external or internal forcing (e.g. a cloud rising above the level at which condensation occurs or a jet with combustion). Laboratory experiments confirm the sensitivity of entrainment to the type of forcing (e.g. Agrawal \& Prasad 2004; Bhat \& Narasimha 1996). The physical reason for the change in this ratio is that progressively more of the momentum flux defined by (3.11) is carried by the turbulent eddies.

When $u_{0}^{2} \geqslant\left\{\Delta U_{1}\right\}_{A}^{2}$, the presence of strong external turbulence controls the outward movement $E_{b}$ of the interface rather than the turbulence of the jet. Here the outer boundary diffuses like a passive interface in a turbulent flow, i.e. $E_{b} \sim \sqrt{\left\langle v_{T}\right\rangle / t}$ where $\left\langle v_{T}\right\rangle \sim u_{0} L_{x}$ and the mean travel time along the jet is $t=x /\left\{\Delta U_{1}\right\}_{A}$ (see (3.4) and table 1).

\section{Application to shear layers}

\subsection{Swirling shear layers}

The physics of unidirectional shear layers has profound implications for shear layers in swirling flows. To illustrate these processes, consider a layer of fluid rotating at an angular velocity $2 \Omega_{0}$ lying between a plane at $x_{3}=0$ and a free surface (or inversion layer) at height $x_{3}=H$. Within a cylindrical region of radius $R_{0}$, intense buoyancy forces are introduced which drive a mean vertical velocity $U_{3}\left(x_{3}\right)$ such that $\partial U_{3} / \partial x_{3}$ is positive over most of the depth of the layer. At the top of the layer the narrow upward plume is arrested and the buoyant fluid spreads out and circulates downwards within a wider circular region whose radius is much greater than $R_{0}$. The vertical straining motion in the core $\left(r<R_{0}\right)$ stretches the background vorticity so that $\Omega_{3}$ is greatly increased (in a laboratory experiment with bubbles $\Omega_{3} / \Omega_{0} \approx 7$, while in hurricanes $\Omega_{3} / \Omega_{0} \sim 10$ ) (Turner 1966; H. J. S. Fernando \& S. A. Smirnov, personal communication). The dynamics of the vortex depend greatly on the ratio $R_{0} / H$.

We consider an idealized case (which could be realized in a laboratory) where $R_{0} / H \gg 1$ and the forcing generates a turbulent recirculating motion. This results in the formation of a forced vortex for $r<R_{0}$ where $\Omega_{3} \approx \Omega_{v 0}$ and $U_{\theta}=\Omega_{v 0} r$. Outside the vortex the strain rate is significant and leads to a greatly reduced mean vorticity outside the vortex core (i.e. at $t=0$, and for $r>R_{0}, \Omega_{3} \ll \Omega_{v 0}$ but $\left.\Sigma_{12}=\partial U_{1} / \partial x_{2}+\partial U_{2} / \partial x_{1} \sim \Omega_{v 0}\right)$. The energy of the turbulence $\overline{u_{i}^{2}}$ is significant where mean strain and mean vorticity are significant at $r \approx R_{0}$ but decreases towards the centre of the vortex. This state of the vortex rapidly evolves. First, this is because the turbulence decays in the forced vortex, a theoretical result from Townsend (1976), 


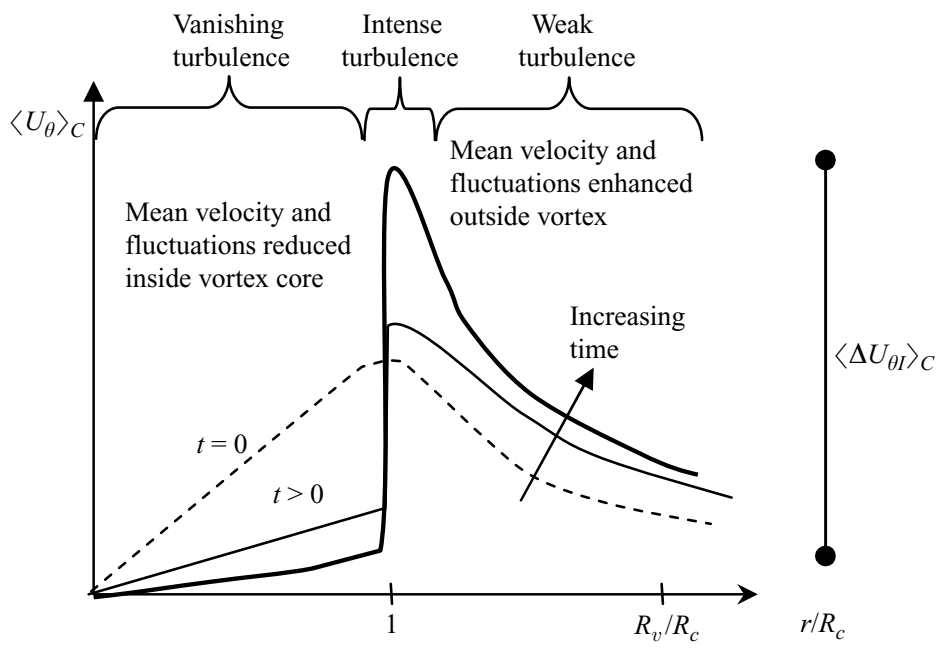

FIGURE 5. Azimuthial velocity in a turbulent hurricane-like vortex, showing how the formation of a turbulent interface at $r=R_{c}$ determines the flow structure. $R_{v}$ is the outer radius.

confirmed by the experiments of Vladimirov (1982). Secondly, turbulence is highly strained and amplified by the mean strain rate $\Sigma_{12}$ near $r=R_{0}$ (Miyazaki \& Hunt 2000; Bassom \& Gilbert 1998). Consequently, as explained in $\S 2$, a sharp interface develops because there is a large radial gradient in turbulence, such that $\partial \overline{u_{i}^{2}} / \partial r \gg \overline{u_{i}^{2}} / R_{0}$. This triggers the amplification of the mean vorticity $\Omega_{3} \approx \partial U_{\theta} / \partial r$. As with the broad class of interfaces discussed in $\S 3.1$, there is also jump in the conditionally averaged azimuthal velocity at the edge of the vortex which is equivalent to a delta-function in the vertical vorticity, i.e. the conditionally averaged vorticity field is $\left\langle\Omega_{3}\right\rangle_{C}=\Omega_{v}(t)\left(1-H\left(r-R_{c}\right)\right)+\left\langle U_{\theta I}\right\rangle_{C} \delta\left(r-R_{c}\right)$, where $R_{c}(t)$ is the core radius which varies with time and $R_{c}=R_{0}$ at $t=0$. Note that $\left\langle U_{\theta}\right\rangle_{C}=\Omega_{v} r$ for $r<R_{c}$ and $\left\langle U_{\theta}\right\rangle_{C}=\left(\Omega_{v} R_{c}+\left\langle\Delta U_{\theta I}\right\rangle_{C}\right) R_{c} / r$ for $R_{c}<r<R_{v}$ (see figure 5).

In a rotating flow, this peak in vorticity significantly affects the peak mean velocity, unlike the case of a planar flow. This is because the total angular momentum is conserved within an outer radius $R_{v}$, i.e. $I=\int_{0}^{R_{v}} r U_{\theta} \mathrm{d} r$ is constant. Since at $t=0$, $I=\frac{1}{3} \Omega_{v 0} R_{0}^{3}+\Omega_{v 0}\left(R_{v 0}-R_{0}\right) R_{0}$, for $t>0, I=\frac{1}{3} \Omega_{v} R_{c}^{3}+\left(\Omega_{v} R_{c}+\left\langle\Delta U_{\theta I}\right\rangle_{C}\right)\left(R_{v}-R_{c}\right) R_{c}$, where in an unbounded horizontal domain $R_{v}$ is increasing slowly with time. The key element of this integral constraint is that the vorticity amplification increases in the shear layer, so that $\left\langle\Delta U_{\theta I}\right\rangle_{C} / \Omega_{v} R_{c}$ grows and $\Omega_{v} / \Omega_{v 0}$ decreases. This process can continue until $\Omega_{v} / \Omega_{v 0}$ tends to zero, but only if the turbulent shear layer at $r=R_{c}$ does not move into the stationary core, i.e. $\mathrm{d} R_{c} / \mathrm{d} t=0$. This surprising flow structure, that is a well-observed characteristic of hurricanes (Bengston \& Lighthill 1982), is possible because in strongly rotating flows $E_{b} /\left\langle\Delta U_{\theta I}\right\rangle_{C} \rightarrow 0$ as outlined in $\S 3.1$. This requires that the thickness of the layer $l$ is great enough that rotation suppresses the turbulent diffusion of vorticity, i.e. $l / R_{c} \geqslant u_{0} /\left\langle U_{0}\right\rangle \sim 1 / 10$. A more detailed study of this problem (to be published) shows that if $E_{b} /\left\langle\Delta U_{\theta I}\right\rangle_{C} \approx 0$ but $\left\langle\Delta U_{\theta I}\right\rangle_{C} \neq 0$ then the jump in the shear stress is also zero, implying that $\partial U_{\theta} / \partial r=0$ at $r=R_{c}$. 'Vortex spin up' caused by sharp shear/turbulence fronts and slantwise convection is a feature of some intense rotating storms that occur at higher lattitudes, where there are no hurricanes (Browning 2004). 


\subsection{Boundary layers}

The outer structure of a turbulent boundary layer is similar to that of a wake (Coles 1956). It is bounded by a fluctuating interface with a conditionally averaged velocity jump $\left\langle\Delta U_{1 I}\right\rangle_{C}$ (though this interface has not yet been studied quantitatively in the same detailed manner as those of jets and wakes). Consequently free-stream turbulence with r.m.s. velocity $u_{0}$ has a similar effect on these flows as for jets and wakes, namely that for $u_{0}<\left\langle\Delta U_{1 I}\right\rangle_{C}$ the exterior turbulence has very little effect because of blocking by the interfacial layer. But when $\left.u_{0}\right\rangle\left\langle\Delta U_{1 I}\right\rangle_{C}$ the whole structure breaks up and lumps of boundary layer vorticity and scalar diffuse into the external flow (Hancock \& Bradshaw 1989) comparable to the plume experiments of Ching et al. (1995). In the interior of the turbulent boundary layer where $h / 5 \leqslant x_{3} \leqslant h / 2$ the integral scale $L_{x}$ is less than the distance $x_{3}$ to the 'wall' at $x_{3}=0$, and therefore since the eddies are not directly affected by the outer interface or the wall, the dynamics are quasihomogeneous. However the eddy transport of turbulent energy by inhomogeneity is a sizeable fraction $(\sim 1 / 2-1 / 3)$ of the rate of production of energy (Townsend 1976). In the presence of large-scale energetic free-stream turbulence these inhomogeneities are even more significant.

The shear-layer analysis of $\S 2.2$ (see also Hunt et al. 2006) also helps to explain the structure of the turbulent boundary layer near to the wall $\left(x_{3} \leqslant h / 5\right)$. First, the wall blocks the normal component of turbulence (i.e. $u_{3}=0$ at $x_{3}=0$ ). This condition is also applicable at the free surface or if the wall moves with the mean flow and 'shear-free' boundary layers. But with a sheared boundary layer, the blocking is affected by the sharp variation in the mean vorticity which reaches a maximum in the viscous sublayer whose thickness $l_{v s}$ is of order $10 v / u_{*}$. This is a similar mechanism to the blocking of free-stream turbulence outside a laminar boundary layer (Jacobs $\&$ Durbin 1998). Note that the sharp maximum in the mean vorticity at this level is maintained by the amplification caused by impinging vortices. The blocking effect implies that the eddy length scale in the turbulent flow above the viscous sublayer is of order $\left(x_{3}-l_{v s}\right)$. This can be confirmed by the weighted two-point cross-correlation $\hat{R}\left(x_{3}, x_{3}^{\prime}\right)=\overline{u_{3}\left(x_{3}\right) u_{3}\left(x_{3}^{\prime}\right)} / \overline{u_{3}^{2}\left(x_{3}^{\prime}\right)}$ for $x_{3}<x_{3}^{\prime}$. It is observed from numerical simulations that $\hat{R} \approx f\left(x_{3} / x_{3}^{\prime}\right)$, but $\hat{R} \rightarrow 0$ where $x_{3} \leqslant l_{v s}$. This sheltering mechanism triggers the bursting phenomena at the top of the viscous sublayer as the fluctuating vertical shear $\partial u_{1} / \partial x_{3}$ builds up (e.g. Blackwelder \& Kaplan 1976).

This mechanism is sensitive to the straining of vorticity by eddies at the top of the viscous sublayer and to the profile of the layer. This provides a quantitative explanation for the reduction of mean shear by the effects of long-chain molecules (Lumley 1973; Ptasinski et al. 2003) and of the perpendicular magnetic field in a conducting medium. This concept of 'blocking' or reduced length scale caused by the viscous sublayer has been used by Launder (2004) to correct Reynolds stress transport models for the effect of lower Reynolds number near a wall. It can be used directly to provide a formula that smoothly and simply matches the viscous sublayer to the log layer namely

$$
\begin{gathered}
U_{1}=\frac{u_{*}}{\kappa}\left[\log \left(\frac{x_{3}-l_{v s}}{v / u_{*}}\right)+B\right], \quad \text { for } x_{3}>l_{v s}, \\
U_{1}=u_{*}\left(x_{3} /\left(v / u_{*}\right)\right), \quad \text { for } x_{3} \leqslant l_{v s}
\end{gathered}
$$

$\kappa(=0.4)$ is von Kármán's constant, $u_{*}$ is the friction velocity and $B$ is a constant (see figure 6). In Prandtl's log-law formulation, $l_{v s}=0$ and there is no smooth matching with the viscous sublayer. The logarithm formula is only correct to about $10 \%$ for 


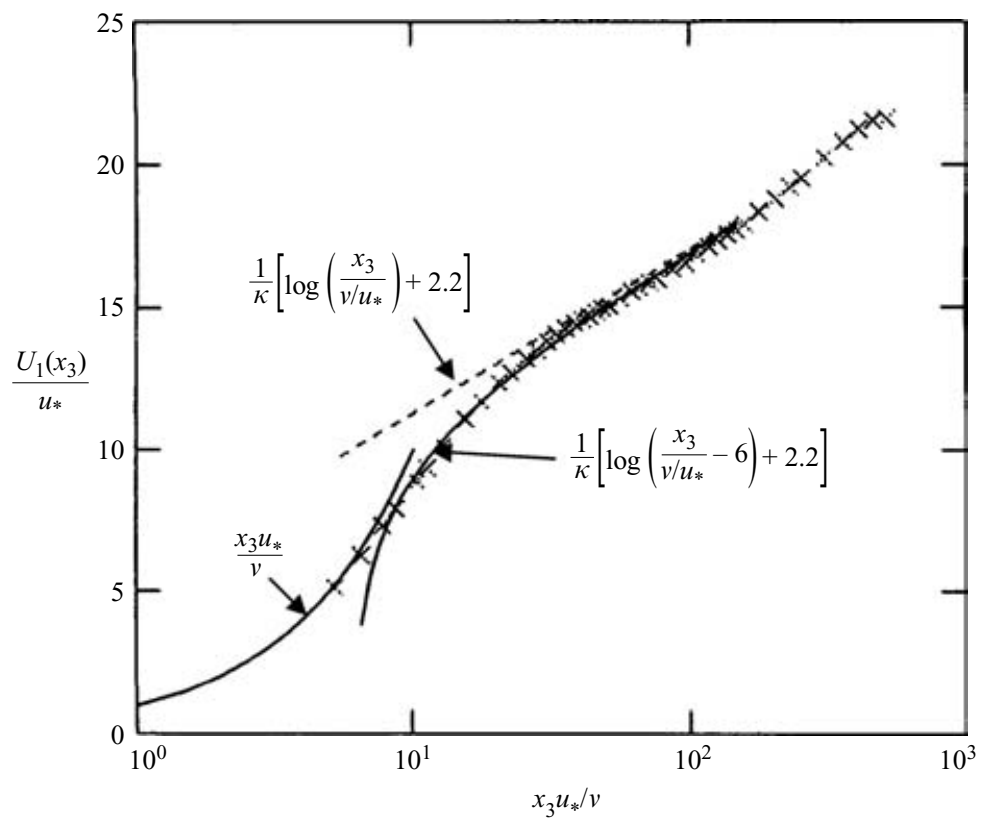

FIGURE 6. Comparison between a displaced log-law formulation (equations (4.1), (4.2)), Prandtl's log-law and experimental measurements (denoted by $\times$ ) of the mean velocity in a turbulent pipe flow at $R e=17500$, where $x_{3}$ is the distance from the pipe wall.

$x_{3} \geqslant 30 v / u_{*}$. Craft et al. (2002) have developed new wall functions which essentially reproduce (4.1) and (4.2) and are demonstrated to provide superior accuracy near walls and global modelling of some flow problems. Figure 6 shows a comparison between the displaced log-law, Prandtl's model and experimental measurements for the mean turbulent flow in a pipe.

The second feature of the turbulence and shearing interaction near the wall concerns the local effect of large inactive eddies generated in the interior of the boundary layer as they impact on the vorticity of the high-shear region (Townsend 1976; Hunt \& Morrison 2000). Over the wavenumber range $h^{-1}<k_{1}<x_{3}^{-1}$ where the energy spectrum of these long eddies is dominated by the effect of mean shear, field experiments and large-eddy simulations and linear theory (Hanazaki \& Hunt 2004; Kader \& Yaglom 1991; Redelsperger, Mahe \& Carlotti 2001) show that the spectrum inside the boundary layer has the general form $E_{i i}\left(k_{1}\right) \sim u_{*}^{2} k_{1}^{-1}$ (for $i=1,2,3$ ). As the eddies impact on the wall, they induce an irrotational blocking flow (see $\S 2.1$ ) so that

$$
E_{11} \sim E_{22} \sim u_{*}^{2} k_{1}^{-1}, \quad E_{33} \sim u_{*}^{2} x_{3}
$$

(Hunt \& Carlotti 2001). The striking point about this spectrum is that the horizontal component of the low-wavenumber turbulent eddies varies slowly with height in the surface layer whereas the mean velocity gradient is varying rapidly (Hogstrom, Hunt \& Smedman 2002). This well-known feature of turbulent boundary layers is used by wind engineers to differentiate between mean and fluctuating loads on tall structures.

The impact of large-scale (or 'inactive') highly elongated eddies on the shear layer tends to amplify the mean vorticity which is consistent with (4.3). This mechanism does not require any nonlinear 'cascade' of large-scale energy to small scales within this shear layer to ensure an equilibrium state for the large scale turbulence. But the 
streamwise length scale of the largest of these eddies, which may be several times greater than the boundary layer thickness, is determined by a nonlinear feedback between the wall shear layers and the outer flow as proposed in several models of the wall-layer dynamics, reviewed by Hunt et al. (2001). By contrast, smaller-scale eddies $\left(k_{1}>x_{3}^{-1}\right)$ are quasi-homogeneous and their equilibrium state is determined by a cascade of energy to smaller scales (Mann 1994).

\section{Concluding remarks}

Whenever turbulence is adjacent to regions where its energy is non-existent, or much weaker, continuous fluctuating interfaces tend to form leading to intense gradients of turbulent energy, mean velocity and concentration of scalars. Similar mechanisms also produce interfaces and amplify mean gradients in thin regions of high shear initiated where turbulence that has been independently generated is present on one or both sides of the interface. In this paper approximate models have been derived to describe these non-diffusive or counter-gradient properties of turbulence near these interfaces. It is shown that they can have wide ranging effects on the overall flow, particularly through controlling the entrainment processes. These mechanisms may be more significant in flows where sharp fronts occur (e.g. in geophysical flows) than where the turbulence dynamics are dominated by inertial and body forces. With the aid of PIV measurement systems and numerical simulations of turbulent flows, it is now possible to measure and compute the geometrical and statistical properties of these interfaces, and the flows associated with them. These studies show that it is essential to focus on the local structures of these very inhomogeneous regions of turbulence to understand and predict many, if not most, kinds of turbulent flow. In these regions this approach is more revealing than focussing on the average Eulerian properties, which effectively smear out intense gradients. This implies that better models for the statistical properties of turbulence are likely to be based on statistical measures defined in relaion to moving interfaces. Local dynamics should be calculated in this frame of reference. Some of the ideas reviewed here might contribute to such an approach, which has some similarities to modern techniques in combustion modelling using probability density functions for flame fronts and regions of intense mixing (Pope 2000).

J.C.R.H. is grateful for support from Cornell University where he is a visiting Mary B. Upson Professor and from the Centre for Polar Observation and Modelling at UCL, which is funded by NERC. The late Professor Frans Nieuwstadt of TU Delft encouraged our study of interfaces over several years. We are grateful to Professor Y. Grachev of Kyiv for organising a NATO workshop in 2004 where some of these ideas evolved, and to Professor Fernando and Dr Smirnov for sharing their preliminary experimental results at Arizona State University.

\section{REFERENCES}

Agrawal, A. \& Prasad, A. K. 2004 Evolution of a turbulent jet subjected to volumetric heating. J. Fluid Mech. 511, 95-123.

Bassom, A. P. \& Gilbert, A. D. 1998 The spiral wind-up of vorticity in an inviscid planar vortex. J. Fluid Mech. 371, 109-140.

Batchelor, G. K. \& Proudman, I. 1954 The effect of rapid distortion of a fluid in turbulent motion. Q. J. Mech. Appl. Maths 7, 83-103.

Batchelor, G. K. 1967 An Introduction to Fluid Dynamics. Cambridge University Press. 
Bengston, L. \& Lighthill, M. J. (Eds.) 1982 Intense Atmospheric Vortices: Proc. Joint IUTAM/IUGG Symp., Reading, UK, July 14-17, 1981. Springer.

BHAT, G. S. \& NARASIMHA, R. 1996 A volumetrically heated jet: large-eddy structure and entrainment characteristics. J. Fluid Mech. 325, 303-330.

Bisset, D. K., Hunt, J. C. R. \& Rogers, M. M. 2002 The turbulent/non-turbulent interface bounding a far wake. J. Fluid Mech. 451, 383-410.

BLACKWELDER, R. F. \& KAPLAN, R. E. 1976 On the wall structure of the turbulence boundary layer. J. Fluid Mech. 76, 89-112.

Bretherton, F. P. \& Turner, J. S. 1968 On the mixing of angular momentum in a stirred rotating fluid. J. Fluid Mech. 32, 449-464.

BRITTER, R. E. \& Simpson, J. E. 1978 Experiments on the dynamics of a gravity current head. J. Fluid Mech. 88, 223-240.

BRowning, K. A. 2004 The sting at the end of the tail: damaging winds associated with extratropical cyclones. Q. J. R. Met. Soc. 130, 375-399.

CARruthers, D. J. \& Hunt, J. C. R. 1986 Velocity fluctuations near an interface between a turbulent region and a stably stratified layer. J. Fluid Mech. 165, 475-501.

Chandrsuda, C., Mehta, R. D., Weir, A. D. \& Bradshaw, P. 1978 Effect of free-stream turbulence on large structure in turbulent mixing layers. J. Fluid Mech. 85, 693-704.

Ching, C. Y., Fernando, H. J. S. \& Robles, A. 1995 Breakdown of line plumes in turbulence environments. J. Geophys. Res. (Oceans) 100, 4707-4713.

Coceal, O. \& Belcher, S. E. 2004 A canopy model of mean winds through urban areas. Q. J. R. Met. Soc. 130, 1349-1372.

Coles, D. 1956 The law of the wake in the turbulent boundary layer. J. Fluid Mech. 1, 191-226.

Corrsin, S. \& Kistler, A. 1955 free-stream boundaries of turbulent flows. Tech. Rep NASA 1244, Washington DC.

Craft, T. J., Gerasimov, A. V., Iacovides, H. \& Launder, B. E. 2002 Progress in the generalization of wall-function treatments. Intl J. Heat Fluid Flow. 23, 148-160.

Craik, A. D. D. 1991 Continous spectrum of Orr-Sommerfeld equation - a note on a paper of Grosch and Salwen. J. Fluid Mech. 226, 565-571.

Davidson, P. A. 2004 Turbulence. Oxford University Press.

Dickens, C. 1865 Our Mutual Friend. Chapman \& Hall.

Durbin, P. A. \& PetTerson Reif, B. 2000 Statistical Theory and Modelling of Turbulent Flows. John Wiley \& Sons.

Gartshore, I. S., Durbin, P. A. \& Hunt, J. C. R. 1983 The production of turbulent stress in a shear flow by irrotational fluctuations. J. Fluid Mech. 137, 307-329.

Gough, D. O. \& LyNDEN-Bell, D. 1968 Vorticity expulsion by turbulence: astrophysical implications of an Alka-Seltzer experiment. J. Fluid Mech. 32, 437-447.

Grachev, Y. \& Hunt, J. C. R. 2006 (Eds.) Flow through Plant Canopies and Urban Terrains. NATO-ASI Summer School. Kluwer.

Grosch, C. E. \& Salwen, H. 1978 The continuous spectrum of the Orr-Sommerfeld equation. Part 1. The spectrum and the eigenfunctions. J. Fluid Mech. 87, 33-54.

HanazaKi, H. \& Hunt, J. C. R. 2004 Structure of unsteady, stably stratified turbulence with mean shear. J. Fluid Mech. 507, 1-42.

HANCOCK, P. E. \& BRADSHAW, P. 1989 Turbulence structure of a boundary layer beneath a turbulent free-stream. J. Fluid Mech. 205, 45-76.

Hodson, H. P. \& Howell, R. J. 2005 Bladerow interactions, transition, and high-lift aerofoils in low-pressure turbines. Annu. Rev. Fluid Mech. 37, 71-98.

Hogstrom, U., Hunt, J. C. R. \& Smedman, A. S. 2002 Theory and measurements for turbulence spectra and variances in the neutral surface. Boundary Layer Met. 103, 101-124.

Holmes, P., Lumley, J. L. \& Berkooz, G. 1996 Turbulence, Coherent Structures, Dynamical Systems and Symmetry. Cambridge University Press.

Hunt, J. C. R. 1985 Turbulent diffusion from sources in complex flows. Annu. Rev. Fluid Mech. 17, 447-485.

Hunt, J. C. R. 1995 Effects of body forces on turbulence. In Advances in Turbulence $V$ (ed. R. Benzi), pp. 229-235. Kluwer.

Hunt, J. C. R. \& CARLotTi, P. 2001 Statistical structure at the wall of the high Reynolds number turbulent boundary layer. J. Flow, Turbulence Combust. 66, 453-475. 
Hunt, J. C. R. \& CARruthers, D. J. 1990 Rapid distortion theory and the 'problems' of turbulence. J. Fluid Mech. 212, 497-532.

Hunt, J. C. R. \& Durbin, P. A. 1999 Perturbed vortical layers and shear sheltering. Fluid Dyn. Res. 24, 375-404.

Hunt, J. C. R., Eames, I., Westerweel, J. \& Robinson, T. 2006 Mechanics of inhomogeneous turbulence and interfacial layers. IUTAM Proc. (submitted).

Hunt, J. C. R. \& Morrison, J. F. 2000 Eddy structure in turbulent boundary layers. Eur. J. Mech. B 19, 673-694.

Hunt, J. C. R., Sandham, N. D., Vassilicos, J. C., Launder, B. E., Monkewitz, P. A. \& Hewitt, G. F. 2001 Developments in turbulence research: a review based on the 1999 programme of the Isaac Newton Institute, Cambridge. J. Fluid Mech. 436, 353-391.

Hunt, J. C. R. \& SAvill, A. M. 2002 Guidelines and criteria for the use of turbulence models in complex flows. In Turbulence Modelling (ed. G. F. Hewitt \& J. C. Vassilicos). Cambridge University Press.

Hunt, J. C. R. \& Vassilicos, J. C. 2002 Turbulent Structure and Vortex Dynamics. Cambridge University Press.

Hussain, A. K. M. F. 1986 Coherent structures and turbulence. J. Fluid Mech. 173, 303-356.

JACOBS, R. G. \& Durbin, P. A. 1998 Shear sheltering and the continuous spectrum of the OrrSommerfeld equation. Phys. Fluids. 10, 2006-2011.

KADER, B. A. \& YAGLOM, A. M. 1991 Spectra and correlation functions of surface layer atmospheric turbulence in unstable thermal stratification. In Turbulence and Coherent Structures (ed. O. Métais \& M. Lesieur). Selected papers from Turbulence 90: Organised Structures and Turbulence in Fluid Mechanics, Grenoble, 18-21 September 1989. Kluwer.

KirchноF, G. R. 1869 Uber die bewegung eines rotationskorpen in ein flussigkeit. Crelle 71, 237.

Kolmogorov, A. N. 1941 Dokl. Akad. Nauk. SSSR. 30, 3201. (English translation, Kolmogorov, A. N. The local structure of turbulence in incompressible viscous fluid for very large Reynolds number. Proc. R. Soc. Lond. 434 (1991), 9-13).

Kovasznay, L. S. G., Kibens, V. \& Blackwelder, R. F. 1970 Large-scale motion in the intermittent region of a turbulent boundary layer. J. Fluid Mech. 41, 283-325.

LAUNDER, B. E. 2004 Lecture at the 80th Birthday of Jean Mathieu. Ecole Central de Lyon.

Lighthill, M. J. 1957 The fundamental solution for small steady three-dimensional disturbances to a two-dimensional parallel shear flow. J. Fluid Mech. 3, 113-144.

Long, R. R. \& Chen, T.-C. 1981 Experimental evidence for the existence of the 'mesolayer' in turbulent systems. J. Fluid Mech. 105, 19-59.

LugT, H. J. 1983 Vortex Flow in Nature and Technology. Wiley.

Lumley, J. L. 1973 Drag reduction in turbulent flow by polymer additives. J. Polymer Sci. 7, 263-290.

Magnaudet, J. 2003 High-Reynolds-number turbulence in shear-free boundary layer: revisiting the Hunt-Graham theory. J. Fluid Mech. 484, 167-196.

Mann, J. 1994 The spatial structure of neutral atmospheric surface-layer turbulence. J. Fluid Mech. 273, 141-168.

Mathew, J. \& Basu, A. J. 2002 Some characteristics of entrainment at a cylindrical turbulence boundary. Phys. Fluids. 14, 2065-2072.

MiYazAKi, T. \& HunT, J. C. R. 2000 Linear and non-linear interactions between a columnar vortex and external turbulence. J. Fluid Mech. 402, 349-378.

Morton, B. R., Taylor, G. I. \& Turner, J. S. 1956 Turbulent gravitational convection from maintained and instantaneous sources. Proc. R. Soc. Lond. 234, 1-23.

Nagata K., Wong, H. Y. W., Hunt J. C. R., SajJadi, S. G. \& Davidson, P. A. 2005 Weak mean flows induced by anisotropic turbulence impinging onto planar and undulating surfaces. $J$. Fluid Mech. (submitted).

Pedley, T. J. 1980 The Fluid Mechanics of Large Blood Vessels. Cambridge University Press.

Phillips, O. M. 1955 The irrotational motion outside a free boundary layer. Proc. Camb. Phil. Soc. 51, 220.

Pope, S. B. 2000 Turbulent Flows. Cambridge University Press.

PRANDTL, L. 1925 Bericht: über Untersuchungen zur ausgebildeten Turbulenz. Z. Angew. Math. Meth. 5, 136-139. 
Ptasinski, P. K., Boersma, B. J., Nieuwstadt, F. T. M., Hulsen, M. A., Van den Brule, B. H. A. A. \& Hunt, J. C. R. 2003 Turbulent channel flow near maximum drag reduction: simulations, experiments and mechanisms. J. Fluid Mech. 490, 251-291.

Redelsperger, J.-L., Mahe, F. \& CArlotti, P. 2001 A simple and general subgrid model suitable both for surface layer and free-stream turbulence. Boundary-Layer Met. 101, 375-408.

Richardson, L .F. 1922 Weather Predictions by Numerical Processes. Cambridge University Press.

Smedman, A. S., Hogstrom, U., Hunt, J. C. R. \& SahlÉe, E. 2006 The forced convection regime of atmospheric surface layer flow, and its implications for the exchange of heat and matter at the surface of the ocean. Q. J. R. Met. Soc. (submitted).

TAYLOR, G. I. 1958 Flow induced by jets. J. Aero. Sci. 25, 464-465.

Townsend, A. A. 1966 The mechanism of entrainment in free turbulent flows. J. Fluid Mech. 26, 689-715.

Townsend, A. A. 1976 The Structure of Turbulent Shear Flows, 2nd Edn. Cambridge University Press.

Turner, J. S. 1966 The constraints imposed on tornado-like vortices by the top and bottom boundary conditions. J. Fluid Mech. 25, 377-400.

Turner, J. S. 1973 Buoyancy Effects in Fluids. Cambridge University Press.

TURNER, J. S. 1986 Turbulent entrainment: the development of the entrainment assumption, and its application to geophysical flows. J. Fluid Mech. 173, 431-471.

VAssilicos, J. C. 2001 (Ed.) Intermittency in Turbulent Flows. Cambridge University Press.

Vladimirov, V. A. 1982 In Intense Atmospheric Vortices: Proc. Joint IUTAM/IUGG Symp., Reading, UK, July 14-17, 1981. Springer.

Warhaft, Z. 2000 Passive Scalars in Turbulent Flows. Annu. Rev. Fluid Mech. 32, 203-240.

Westerweel, J., Hofmann, T., Fukushima, C. \& Hunt, J. C. R. 2002 The turbulent/non-turbulent interface at the outer boundary of a self-similar turbulent jet. Exps. Fluids 33, 873-878.

Westerweel, J., Pedersen, J. M., Fukushima, C. \& Hunt, J .C. R. 2005 The turbulent/nonturbulent interface of a self-similar turbulent jet. Phys. Rev. Lett. 95, 199902.

Wong, H. Y. W. 1985 Shear-free turbulence and secondary flow near angled and curved surfaces. $\mathrm{PhD}$ Thesis, Cambridge University.

Wu, X., Jacobs, R. G., Hunt, J. C. R. \& Durbin, P. A. 1999 Simulation of boundary layer transition induced by periodically passing wakes. J. Fluid Mech. 398, 109-153. 